\title{
Genetics of egg production in Drosophila sechellia
}

\author{
CD Jones \\ Department of Biology, University of Rochester, Rochester, NY 14627, USA
}

\begin{abstract}
Drosophila sechellia, an island endemic that specializes on a single host plant, has a lower rate of egg production than its generalist sister species $D$. melanogaster, $D$. simulans, and $D$. mauritiana. Earlier work showed that part of this difference in egg production was due to a reduction in the number of ovarioles in $D$. sechellia relative to its sister species. Here, I extend this earlier work by genetically analyzing the difference in egg production between $D$. sechellia and $D$. simulans. In all, 10 genetic markers were used in several interspecific backcrosses to identify chromosome regions that affected the rate of egg production. While previously mapped factors affecting ovariole number appear to impact
\end{abstract}

the rate of egg production, new, non-ovariole factors were also identified. Overall, the difference in egg production between $D$. sechellia and $D$. simulans appears to be a polygenic trait. The relationship between these factors and genes involved the adaptation of $D$. sechellia to its host plant is not yet clear. The data are consistent with the hypothesis that decline in egg production is, in part, a negative pleiotropic effect of genetic changes required for host specialization in $D$. sechellia, although finer-scale genetic analysis of both traits is needed to truly test this hypothesis. Heredity (2004) 92, 235-241, advance online publication, 7 January 2004; doi:10.1038/sj.hdy.6800401

Keywords: oviposition; egg production; species differences; Drosophila; Morinda; cost of resistance

\section{Introduction}

An island endemic of the Seychelles, Drosophila sechellia almost exclusively uses the fruit of Morinda citrifolia (Morinda) as its host (Louis and David, 1986). To use this host, D. sechellia has evolved a strong preference for and resistance to the toxins in Morinda (Louis and David, 1986; R'Kha et al, 1991; Jones, 2004). D. sechellia also appears to have evolved a slower rate of egg production than its sister species after it split from its presumably D. simulans-like ancestor (Coyne et al, 1991; R'Kha et al, 1997). Given that egg production is closely related to female fitness in Drosophila (Boulétreau-Merle et al, 1982), the persistence of a low rate of egg production in $D$. sechellia is surprising. One possible explanation for the low rate of egg production is that it is a deleterious pleiotropic effect of genes involved in specializing on Morinda.

Mechanistically, the lower production of D. sechellia is partially explained by the fact that it has half as many ovarioles as its closest relatives (Coyne et al, 1991). Hodin and Riddiford (2000) have shown that this difference in ovariole number between $D$. sechellia and D. melanogaster ovaries is due to slower ovarian growth rates in $D$. sechellia at embryonic and first instar stages and at the later third instar stage. Coyne et al (1991), using four genetic markers in a series of interspecific crosses between $D$. simulans and $D$. sechellia, showed that chromosomes 2 and 3 both harbored genetic factors affecting ovariole number. The $X$ chromosome had no effect.

Correspondence: $C D$ Jones, Center for Population Biology, University of California, Davis, Davis, CA 95616, USA. E-mail: cojo@ucdavis.edu Received 30 December 2002; accepted 20 September 2003
Ovariole number alone, however, does not fully explain the lowered egg production in D. sechellia ( $\mathrm{R}^{\prime} \mathrm{Kha}$ et al, 1997). R'Kha et al showed that there was no strong correlation between maximum daily fecundity and ovariole number in D. sechellia, although there was evidence for this relationship in D. simulans. Furthermore, they showed that the rate of egg production of $D$. sechellia standardized by ovariole number was only $60 \%$ of the $D$. simulans rate. The genetics of lower rate of egg production in $D$. sechellia clearly involves more genes than those involved in the interspecific difference in ovariole number.

To determine the relationships between the lowered rate of egg production in $D$. sechellia and genes affecting ovariole number $v s$ other types of genes, and to look at how these two classes of factors interact, I coarsely mapped genetic factors affecting the low rate of egg production in $D$. sechellia. These data will provide an insight into the genetic complexity of interspecific differences in egg production and will also provide a foundation for future fine-scale genetic analyses of egg production.

\section{Materials and methods}

\section{Stocks}

The fly stocks used are presented in Table 1. All flies were reared at low density on agar-yeast-cornmeal media at $24^{\circ} \mathrm{C}$.

\section{Crosses}

Visible mutations were used as genetic markers to map factors affecting egg production. Figure 1 diagrams a typical interspecific backcross analysis using dominant and recessive markers. Recessively marked $D$. sechellia 
Table 1 Species and strains used

\begin{tabular}{|c|c|c|}
\hline Species & Stock & Comment \\
\hline D. mauritiana & Synthetic & $\begin{array}{l}\text { Mixture of six isofemale lines collected by O Kitagawa in Mauritius in } 1981 \text { and pooled in 1983. From J } \\
\text { Coyne }\end{array}$ \\
\hline D. melanogaster & OR-R & From Bloomington Drosophila Stock Center \\
\hline \multirow[t]{6}{*}{ D. sechellia } & Line 1 & 'Roberstson' collected from Seychelles in 1981 by Tsacas and Bachli. Wild-type isofemale line \\
\hline & Line $4,24,81$ & Collected in the Seychelles by JR David in 1985 \\
\hline & sy007, sy035 & Collected in the Seychelles by B Ballard in 1998 \\
\hline & jen pur & From J Coyne \\
\hline & $z n f ; j c n$ & Constructed from stocks provided by J Coyne \\
\hline & $z n v f$ & From J Coyne \\
\hline \multirow[t]{5}{*}{ D. simulans } & Islamorada & Wild type collected in Islamorada, FL \\
\hline & Antp/Serr & Constructed from stocks provided by J Coyne and D Presgraves \\
\hline & $\mathrm{Cy} / \mathrm{Cy}$ & From Species Stock Center \\
\hline & $\mathrm{Dl} / \mathrm{Ubx}$ & From J Coyne \\
\hline & f; nt pm; ste & From J Coyne \\
\hline
\end{tabular}

D. simulans

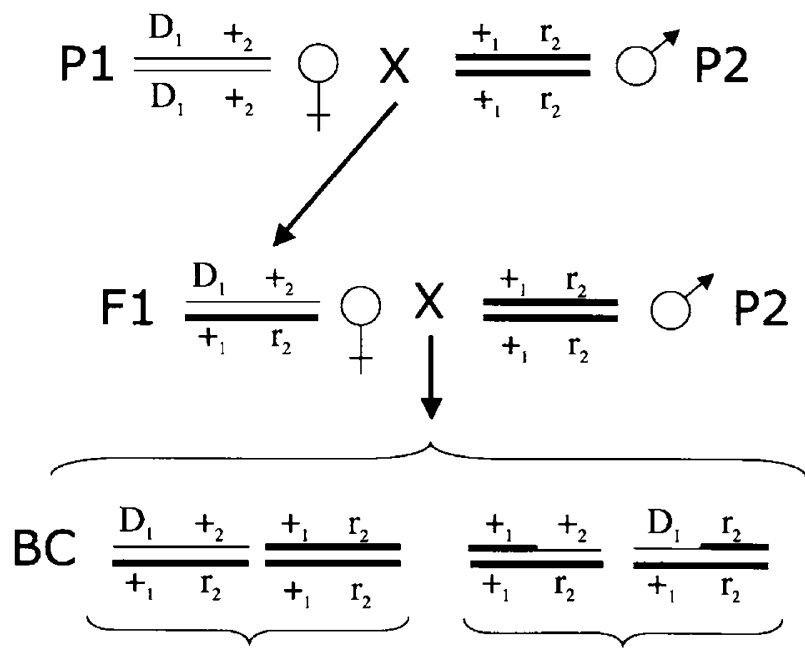

Parental-like genotypes

Figure 1 Diagram of a typical interspecific backcross (BC) with two markers. $D_{1}$ represents a homozygous viable dominant marker in $D$. simulans. $r_{2}$ represents a recessive marker in $D$. sechellia. Recessively marked $D$. sechellia males were mated to dominantly marked $D$. simulans females. The resulting F1 females were backcrossed to males from the $D$. sechellia marker strain. Recessive markers identify chromosome regions that are homozygous for material from $D$. sechellia. Dominant and wild-type markers identify regions heterozygous for material from $D$. sechellia and $D$. simulans. On average, three-fourths of the genes in the BC progeny come from $D$. sechellia and one-fourth come from $D$. simulans.

males were mated to dominantly marked $D$. simulans females. The resulting F1 females were backcrossed to males from the $D$. sechellia marker strain. On average, three-fourths of the genes in these backcross progeny come from $D$. sechellia and one-fourth come from $D$. simulans. Recessive markers identify chromosome regions that are homozygous for material from $D$. sechellia. Wild-type or dominant markers identify regions heterozygous for material from $D$. sechellia and $D$. simulans. Owing to recombination in F1 females, markers only indicate the species origin of chromosome regions, and not of entire chromosomes. The Results section contains additional details about each cross.

\section{Egg production assay}

Egg production per ovariole has been shown to increase when $D$. sechellia was able to oviposit on media containing Morinda fruit or aliphatic acids common in this fruit ( $\mathrm{R}^{\prime} \mathrm{Kha}$ et al, 1997; Amlou et al, 1998). Conversely, these same compounds usually inhibit egg production in $D$. simulans. These facts complicate any mapping of factors affecting egg production in hybrids between these species. To prevent the confounding of genes for preference and genes for egg production, I presented females with both types of media: one untainted and one tainted with octanoic acid. Octanoic acid is the most abundant aliphatic compound in Morinda fruit (Farine et al, 1996). Octanoic acid is weakly volatile and has been shown to strongly affect the ovipositioning behavior of $D$. sechellia when the flies are presented a choice between media with octanoic acid and media without octanoic acid (Amlou et al, 1998). Media were prepared using Drosophila Instant Medium (Carolina Biological Supply Co). Tainted media were $0.07 \%$ octanoic acid by weight (Sigma Chemical Co).

Each female was allowed to oviposit for 2 days in a chamber containing both types of media, after which the total number of eggs laid was scored. To ensure that females had ample uncrowded oviposition substrate available, females were shifted to a fresh set of media and allowed to oviposit for 2 more days. The total number of eggs was again counted. The total for all 4 days was used in the subsequent analyses. All assays were conducted at $20^{\circ} \mathrm{C}$ in a constant-temperature room with relative humidity between 50 and $70 \%$.

\section{Statistics}

As appropriate, either a $t$-test or an analysis of variance (ANOVA) was used (Statview and SAS, SAS Institute, Cary NC). For markers M[1], M[2], ..., M[n], the model statement used for ANOVAs was typically of the standard form, EGGRATE $=\mu+\mathrm{M}[1]+\cdots+\mathrm{M}[n]+\mathrm{M}[1]$ $\mathrm{M}[2] \cdots \mathrm{M}[n-1] \mathrm{M}[n]+\varepsilon$. Simpler models were used if adding parameters failed to improve the fit of the model to the data. ANOVA assumes homoscedacity of the variances. The egg production data produced by this study unfortunately violate this assumption. Specifically, the variance in egg production increases with mean egg production. Fortunately, a straightforward log 
transformation restores homoscedacity to the variances in this case. All ANOVAs, therefore, are preformed on log transformed egg production data.

\section{Results}

\section{Within $D$. sechellia comparison}

I compared the egg production rate among several wild type and mutant marked lines of $D$. sechellia. The results are presented in Table 2 . In all cases egg production was quite low (average across lines 9.14 \pm 0.72 SE). Furthermore, the egg production of flies bearing markers was not significantly different from that of those without markers $(t=0.2725 ; P=0.79)$.

\section{Between-species comparison}

I contrasted the rate of egg production in $D$. sechellia to that of its close relatives, D. melanogaster, D. mauritiana, and $D$. simulans (Figure 2). Isofemale lines of $D$. melanogaster and $D$. simulans both have a high rate of egg production and did not significantly differ from one another $(t=0.159 ; P=0.87)$. The single isofemale line of $D$. mauritiana tested, on the other hand, lays significantly fewer eggs than either $D$. melanogaster or $D$. simulans ( $D$. mauritiana vs $D$. melanogaster; $t=6.30$; $P<0.001)$. D. sechellia, however, lays the fewest $(D$. sechellia os $D$. mauritiana; $t=2.25 ; P=0.029)$. These results are similar to those of $\mathrm{R}^{\prime} \mathrm{Kha}$ et al (1997).

These data further show that the difference in egg production between $D$. sechellia and its sister species is

Table 2 Within $D$. sechellia comparison

\begin{tabular}{lcc}
\hline Isofemale line & Number tested & Egg production (SE) \\
\hline Line 1 & 48 & $8.0(1.0)$ \\
Line 4 & 40 & $8.6(0.7)$ \\
Line 24 & 47 & $9.3(0.6)$ \\
Line 81 & 39 & $10.9(0.6)$ \\
zn f & 52 & $6.6(0.9)$ \\
$j$ cn pur & 54 & $11.3(0.6)$ \\
\hline
\end{tabular}

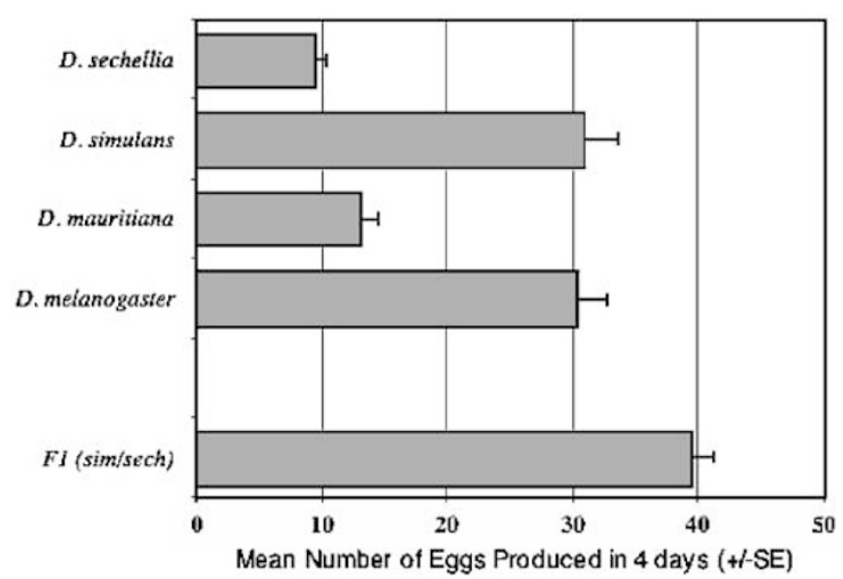

Figure 2 Comparison of egg production rates for $D$. sechellia, $D$. simulans, D. mauritiana, D. melanogaster, and F1 hybrids between $D$. sechellia and D. simulans. Single isofemale lines were tested from each species. All lines were tested simultaneously. Standard error bars are shown. affected by factors other than those that affect ovariole number. Coyne et al (1991) showed that D. sechellia has half as many ovarioles as $D$. simulans. At the same rate of production per ovariole, doubling the number of ovarioles would presumably double $D$. sechellia's egg production. Even after this doubling, however, $D$. sechellia still must increase its egg production by an additional $65 \%$ to be on par with $D$. simulans ( $\mathrm{R}^{\prime} \mathrm{K}$ ha et al, 1997).

\section{Dominance}

I crossed $D$. simulans forked $(f ; 1-56)$, net (nt; 2-0), plum (pm; 2-103), scarlet (st; 3-46), ebony (e; 3-59) females to $D$. sechellia Line 1 males and measured the rate of egg production of the resulting F1 hybrids. As shown in Figure 1, these hybrids have a very high rate of egg production. The fact that F1 hybrids appear to have a $20 \%$ higher rate than pure $D$. simulans is likely due to masking of mildly deleterious alleles in the inbred parental lines. This suggests that the low rate of production of $D$. sechellia is recessive to the high rate of $D$. simulans. This result is confirmed in subsequent backcross analyses (see below).

\section{Multi-chromosome analysis}

To determine which chromosomes harbored factors affecting egg production, I crossed $D$. simulans Ultrabithorax (Ubx; 3-60)/Delta (Dl; 3-66) females to $D$. sechellia zinfindel $(z n ; 1-25)$, forked $(f ; 1-56), j(j ; 2-48)$, cinnabar (cn; 2-67) males. F1 hybrid females harboring $U b x$ were then backcrossed to $D$. sechellia $z n$ f; $j$ cn males. This cross results in 32 different genotypes; however, I only assayed the eight genotypes roughly corresponding to whole chromosome substitutions.

From Figure 3 it is clear that the most D. sechellia-like genotype has the lowest rate of egg production and that the most $D$. simulans-like genotype has the highest rate of egg production as expected. Table 3 presents the results of ANOVA on transformed data (see Materials and methods for a discussion of the transformation). All three of the major chromosomes appear to affect the rate of egg

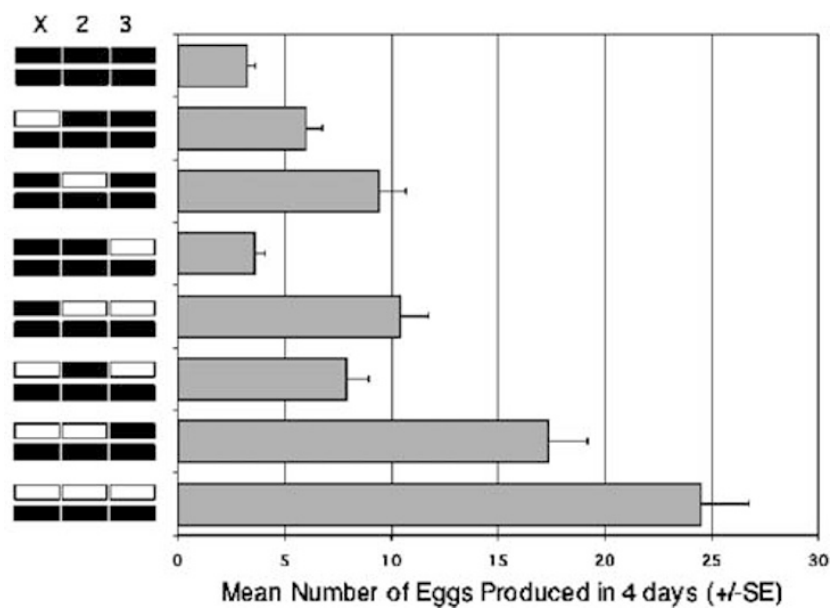

Figure 3 Chromosome 2 has a large effect in multi-chromosomal analysis of egg production. Dark bars on the $Y$-axis represent $D$. sechellia chromosome regions. Light bars represent $D$. simulans chromosome regions. Standard error bars are shown. 
Table 3 ANOVA results: whole chromosome

\begin{tabular}{lrrrr}
\hline Genotype & DF & Mean square & F-value & P-value \\
\hline X & 1 & 11.339 & 52.663 & $<0.0001$ \\
C2 & 1 & 25.624 & 119.007 & $<0.0001$ \\
C3 & 1 & 0.943 & 4.378 & 0.0369 \\
X $\times$ C2 & 1 & 0.004 & 0.018 & 0.8928 \\
X 33 & 1 & 0.241 & 1.121 & 0.2903 \\
C2 $\times$ C3 & 1 & 0.005 & 0.023 & 0.8793 \\
X $\times$ C2 $\times$ C3 & 1 & 0.008 & 0.039 & 0.8441 \\
Residual & 462 & 0.215 & & \\
\hline
\end{tabular}

Model: $\quad$ EGGRATE $=\mu+\mathrm{X}+\mathrm{C} 2+\mathrm{C} 3+\mathrm{X} \times \mathrm{C} 2+\mathrm{X} \times \mathrm{C} 3+\mathrm{C} 2 \times \mathrm{C} 3+\mathrm{X} \times$ $\mathrm{C} 2 \times \mathrm{C} 3+\varepsilon_{X \mathrm{C} 2 \mathrm{C} 3}$.

production. However, the second chromosome clearly has the greatest effect $(53 \%$ of difference between extreme genotypes explained), followed by the $X$ chromosome $(35 \%)$, and then the third $(10 \%)$. No significant interactions were found, suggesting little to no epistasis between these factors.

Interestingly, the most $D$. sechellia-like genotype has a lower rate of egg production than pure $D$. sechellia, whereas the most $D$. simulans-like genotype is roughly the same as that of pure $D$. simulans. There are four possible explanations for this. First, the most likely explanation of this effect is the fact that different (although related) strains were used in this cross than in the pure species controls. As seen in Table 2, there is considerable within-species variation in egg production. Second, both the most $D$. sechellia-like genotype and the most $D$. simulans-like genotype harbor mutant markers that could affect egg production (mutant markers, however, were shown not to strongly affect egg production in pure D. sechellia). Third, this effect could be due to weak linkage between the genetic markers used and the egg production genes. This is unlikely because, if there was weak linkage, I would expect a decrease in egg production for the most $D$. simulans-like genotype, and an increase in egg production of the more $D$. sechellia-like genotype. As this is not the case, my markers must be partially linked to most of the egg production factors. Fourth, given that hybrids between $D$. sechellia and D. simulans exhibit some hybrid breakdown (Joly et al, 1997), one might worry that the differences in egg production among genotypes are the result of hybrid incompatibility. The flies described above have 50-100\% of their genes from $D$. sechellia, depending on their genotype. If hybrid breakdown was playing an important role in determining the rate of egg production, then the most 'hybrid' genotype - that is, the one with the fewest $D$. sechellia genes - should have the lowest rate of egg production. This is clearly not the case as flies with the most $D$. sechellia-like genotypes have the lowest rate of egg production, whereas flies with the most 'hybrid' genotype have the highest rate of egg production.

\section{Analysis of the $X$ chromosome}

I reanalyzed the effect of the $X$ chromosome using an additional marker. This gives me more power to detect an effect if the factor resides in the center of the $X$ chromosome. I crossed $D$. simulans Islamorada females to D. sechellia zn vermillion $(v ; 1-40) f$ males. F1 females were then backcrossed to $D$. sechellia $z n v f$ males. Although this cross generates a variety of genotypes, I tested only the two most extreme. The $X$ strongly affects egg production as noted above in the multi-chromosomal analysis (D. sechellia $\mathrm{X}$ : $N=28$; mean egg production $=6.75 ; \quad D$. simulans $\mathrm{X}: N=40 ;$ mean egg production $=24.45 ; t$-test, $t=6.91 ; P<0.0001)$.

\section{Analysis of chromosome 2}

The multi-chromosome analysis showed that chromosome 2 strongly affects egg production. To coarsely map these egg production factors, I performed two additional crosses. First, I verified the effect of this chromosome by crossing $D$. simulans $U b x / D l$ to $D$. sechellia $j$ cn purple (pur; 2-110). I only assayed those progeny whose second chromosome had all $D$. sechellia markers or all $D$. simulans markers. Not surprisingly, this analysis showed that chromosome 2 significantly affects egg production (D. sechellia genotype: mean egg production $=18.1$, $N=44 ; D$. simulans genotype: mean egg production $=$ 32.6, $N=46 ; t=5.09, P<0.001)$

The whole chromosome analysis of egg production showed, in contrast to the ovariole data of Coyne et al (1991), that the effect of chromosome 2 was greater than that of chromosome 3 . This suggests that chromosome 2 harbors both genes affecting ovariole number and other genes affecting egg production. To map egg production factors within this chromosome, I crossed D. simulans $C y$ (2-6) to D. sechellia cn pur. Hybrid F1 females were backcrossed to $D$. sechellia cn pur males. The cross produces eight genotypes and genetically marks both the center and tips of chromosome 2 .

The results of this analysis are summarized in Figure 4 and Table 4. Only the region linked to $c n$ has a main effect on egg production. In all, $78 \%$ of the effect of chromosome 2 on egg production can be explained by this region. There is also a significant interaction between $C y$ and $c n$. This suggests that there is epistasis between the egg production factor linked to $c n$ and some factor on $2 L$. This interaction explains some of the remaining effect of this chromosome, although some of the unexplained effect may be due to a few factors of small effect that were missed by this analysis.

The marker $c n$ used above is equivalent to the marker that Coyne et al (1991) showed was linked to a factor affecting ovariole number. This suggests, of course, that a large part of the effect of this chromosome is due to this ovariole factor. However, a couple of additional observations suggest that the effect of chromosome 2 is more than an increase in ovariole number. First, Coyne et al (1991) did not detect an interaction between $c n$ and the left arm of the chromosome. This may suggest that there is a nonovariole factor weakly linked to this region. Second, the effect of this chromosome on total egg production is much greater than that expected by an increase in ovariole number alone. Together these data imply that ovariole and nonovariole factors are contributing to the effect of this chromosome. Moreover, it seems likely that both types of factors are linked to $c n$.

\section{Analysis of chromosome 3}

Coyne et al (1991) showed that chromosome 3 had the greatest effect on ovariole number. Not surprisingly, the whole chromosome analysis showed that chromosome 3 


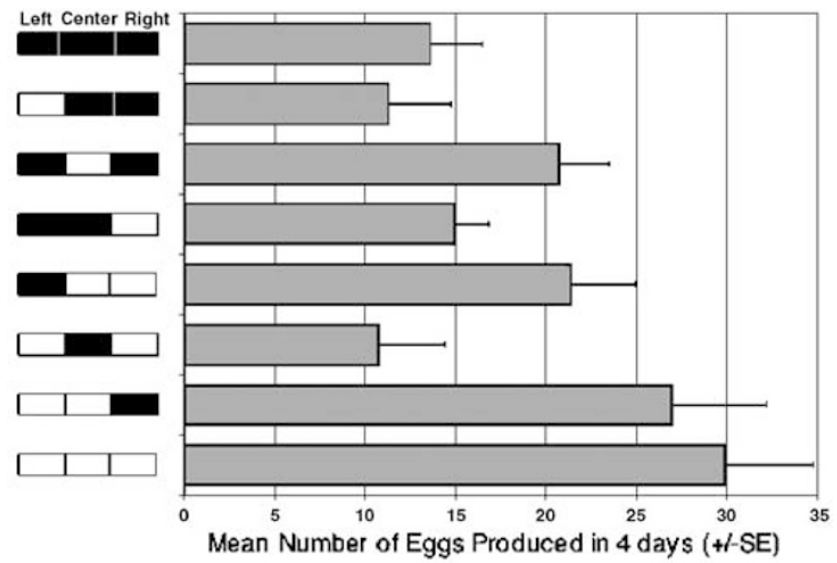

Figure 4 Center of chromosome has a large effect in within chromosome 2 analysis of egg production. Dark bars on the $Y$-axis represent $D$. sechellia chromosome 2 regions. Light bars represent $D$. simulans chromosome 2 regions. Standard error bars are shown.

Table 4 ANOVA results: - chromosome 2

\begin{tabular}{lrcrr}
\hline Genotype & DF & Mean square & F-value & P-value \\
\hline$C y$ & 1 & 0.005 & 0.032 & 0.8579 \\
$c n$ & 1 & 5.028 & 33.647 & $<0.0001$ \\
$p r$ & 1 & 0.020 & 0.133 & 0.7160 \\
$C y \times c n$ & 1 & 0.857 & 5.734 & 0.0174 \\
$C y \times p r$ & 1 & 0.003 & 0.021 & 0.8859 \\
$c n \times p r$ & 1 & 0.005 & 0.034 & 0.8537 \\
$C y \times c n \times p u r$ & 1 & 0.033 & 0.220 & 0.6395 \\
Residual & 246 & 0.149 & & \\
\hline
\end{tabular}

Model: $\quad$ EGGRATE $=\mu+C y+c n+p r+C y \times c n+C y \times p r+c n \times p r+C y \times$ $c n \times p r+\varepsilon_{\text {Cycnpr }}$

affects egg production. Both analyses, however, utilized only one marker. Coyne et al used $e$ (3-59) and I used $U b x$ (3-60). Ubx, in D. simulans, is associated with a small inversion on the right side of the centromere of chromosome 3 (Coyne and Sniegowski, 1994). This inversion weakly suppresses recombination and, as a result, $U b x$ marks a fairly large section of the center of chromosome 3 (Jones and Orr, 1998). The left and right ends of this chromosome were not marked.

To determine if the egg production factor(s) was to the left or right of $U b x$, I contrasted the linkage of the egg production factor(s) to $\mathrm{Dl}$ (3-66) vs Ubx. First, I crossed $D$. simulans $U b x / D l$ to $D$. sechellia Line 1 males. F1 hybrid females were backcrossed to $D$. sechellia Line 1 males. I assayed the egg production of the resulting progeny, which had three genotypes: $U b x /+, D l /+$, and $+/+$ (where + is from D. sechellia). Both $U b x$ and $D l$ are linked to the egg production factors $(U b x /+[N=224]$ vs $+/+[N=221]: t=3.43, P<0.001 ; D l /+[N=231]$ vs $+/+: t=3.47, P<0.001)$. Neither $U b x$ nor $D l$ is substantially more closely linked to the egg production factors (Ubx vs Dl: $t=0.621, P=0.54$ ).

To test the effect of the distal end of the right arm of chromosome 3, I mapped egg production using the marker Serrate (Serr; 3-115). This marker is linked to egg production (Serr $/+[n=29]$ vs $+/+[n=29]: t=3.36$, $P=0.002$ ). The mean difference between Serr and wild type is 8.8 eggs. In contrast, the difference between $U b x$ and wild type and $D l$ and wild type are 4.5 and 5.3 eggs, respectively. This suggests that two egg production factors may reside on this chromosome: one linked to $\mathrm{Ubx} / \mathrm{Dl}$ and another closer to Serr. Unfortunately, about half of the left arm of chromosome 3 is not linked to any of my markers. Any factors in this region of chromosome 3 would not be detected.

\section{Discussion}

Here I have shown that all major chromosomes harbor factors affecting egg production. The second chromosome has the greatest effect, followed by the $\mathrm{X}$ chromosome, and then the third chromosome. The dot fourth chromosome, which contains less than $2 \%$ of the genome, was not tested. The effect of the second chromosome is primarily due to genes near the centromere. The effect of chromosome 3 , on the other hand, is partially due to factors on the right arm of this chromosome. These data suggest that egg production is a somewhat polygenic trait. Egg production, however, is not highly polygenic as the impact of a chromosome on egg production is not proportional to the number of genes on that chromosome. For example, the $\mathrm{X}$ chromosome has the fewest genes of any of the major chromosome elements (17\% of genes, Adams et al, 2000), yet it has the second greatest effect on egg production. Likewise, the right arm of the third chromosome has the most genes of any major chromosome arm $(\sim 25 \%)$ but it has only a weak effect on egg production. (This effect is probably not due to weak linkage between my markers and the egg production genes as I can recover nearly pure species-like egg production.)

Genetics ovariole number vs other egg production factors As noted earlier, the present analysis maps all factors affecting egg production, including the ovariole factors found by Coyne et al (1991). Not surprisingly, there is overlap between the regions identified here and those identified in the earlier analysis. In particular, both the second chromosome and the third chromosome affect egg production as well as ovariole number. Nonetheless, genes affecting ovariole number do not explain the entire egg production difference between $D$. simulans and $D$. sechellia. Furthermore, the overall genetic of these two traits, while related, appear distinct. The $\mathrm{X}$ chromosome affects egg production but does not affect ovariole number. While the second and third chromosomes affect both traits, the second has a greater effect on egg production than the third, which is the opposite of what Coyne et al (1991) showed for ovariole number. The dominance of $D$. sechellia-like ovariole number is roughly additive, whereas the dominance of $D$. sechellia-like egg production is mostly recessive. Some of these differences may be due to the particular marker lines used and the direction of the crosses. These differences, however, are not because different regions were genetically marked in the two studies. I used either the same markers as Coyne et al (1991) (eg cn), markers close to Coyne et al's markers (eg $C y, U b x / D l)$, or crosses that tested intervals containing the regions around Coyne et al's markers (eg the $\mathrm{X}$ chromosome).

There is evidence for epistasis between regions known to harbor ovariole genes and regions containing other types of egg production genes. The region near $\mathrm{cn}$ on the 
second chromosome clearly affects both phenotypes and has a nonadditive interaction with the region near the marker Cy. Coyne et al (1991) suggested that the left arm of chromosome 2 did not affect ovariole number. This epistasis suggests some interconnection between ovariole factors and other genes affecting egg production. This type of interaction, however, does not appear to be common as no other epistatic interactions were seen within or between chromosomes.

\section{Evolution of egg production in $D$. sechellia}

Why $D$. sechellia evolved a lower rate of egg production is not known. There are at least three possible explanations: (1) lower egg production is an adaptation to the ecology of the Seychelles; (2) it is the legacy of past population bottlenecks; or (3) it is a pleiotropic effect of genes involved in other adaptations.

The lower rate of egg production of $D$. sechellia may be an adaptation to specializing on Morinda fruit in the Seychelles. In Hawaiian Drosophila, egg production appears to be related to the availability and quality of hosts with specialists typically having lower rates of egg production (Kampysellis and Heed, 1971). Thus, $D$. sechellia's rate of egg production may be an adaptation to specializing on Morinda. For instance, one might speculate that $D$. sechellia provisions its eggs differently now that it is a host specialist and that this affects the rate of egg production. However, D. mauritiana also has fewer ovarioles and a lower rate of egg production than $D$. simulans and D. melanogaster but is not a host specialist. This suggests that a decline in ovariole number and in the rate of egg production need not be associated with host specialization (although I cannot rule out that both species have evolved a lower rate of egg production as a result of becoming island endemics).

The ancestors of $D$. sechellia were presumably blown from Madagascar or the east coast of Africa to the Seychelles Islands (Lachaise et al, 1988). This small founding population may have had a low rate of egg production and extant $D$. sechellia may simply reflect this fact. David (1979) shows that flight is impaired in gravid females with many ovarioles, which suggests that those flies most likely to found a population on the Seychelles were those with fewer ovarioles. Consistent with this idea, D. mauritiana, the other island endemic, has fewer ovarioles than D. simulans and D. melanogaster. This scenario, however, does not explain why the per ovariole rate of egg production has also declined in D. sechellia.

Alternatively, the small effective population size of $D$. sechellia may have allowed it to accumulate a number of deleterious mutations leading to a general decline in egg production (Cariou et al, 1990). Indeed Kliman et al (2000) have shown that D. sechellia has accumulated many more presumably deleterious unpreferred codon changes than its sister species. D. mauritiana, however, does not show this pattern. Moreover, given that egg production is an important life history trait, egg production is likely under strong selection to remain high if higher egg production is beneficial. It is also formally possible that egg production in $D$. sechellia was historically even lower than it is today and that the rate of egg production has been steadily recovering in $D$. sechellia.
The reduced egg production in D. sechellia may instead be a pleiotropic effect of genes involved in its adaptation to Morinda. Earlier studies suggest that all major chromosomes harbor factors related to the ability of D. sechellia to use Morinda (reviewed in Jones 2004). The pleiotropic effects of these factors could result in a lowered egg production in $D$. sechellia. The $X$ chromosome, for example, strongly affects egg production and also harbors factors affecting adult resistance to the Morinda and larval morphology (Jones, 1998; Sucena and Stern, 2000). Similarly, chromosomes 2 and 3 harbor both resistance factors and egg production factors (Jones, 1998, 2001). The rank order of effects, however, differs between the phenotypes. For instance, the third chromosome consistently has the largest effect on resistance but the weakest effect on egg production, although it does have a large effect on ovariole number (similar genetic markers were used in all three studies). In sum, I cannot reject the pleiotropy hypothesis, although, at the resolution of the present study, my power to test this hypothesis is very limited.

\section{Candidate genes}

Much more work is needed to identify the genes involved in the difference in egg production between $D$. simulans and $D$. sechellia. The genes related to specializing on Morinda are obvious candidates if indeed the decline in egg production is a pleiotropic effect of these genes. Similarly, ovo (also known as shaven-baby) has been shown to be involved in a $D$. sechellia specific difference in larval morphology (Sucena and Stern, 2000). In D. melanogaster, ovo is known to play a role in oogenesis and is important for subsequent embryonic germline development. This gene is located on the $X$ chromosome near the marker $z n$ used in the present study. In future, mapping experiments dissecting the effect of the $X$ chromosome on egg production in $D$. sechellia could be used to determine if the chromosome region around ovo is associated with the egg production phenotype.

Recently, Wayne et al (1997; 2001), Wayne and Mackay (1998), and Wayne and McIntyre (2002) used a combination of QTL mapping, deficiency mapping, and microarray experiments to identify several candidate genes affecting quantitative variation for ovariole number in $D$. melanogaster. These factors reside in two regions of chromosome 3: cytological bands 87 and 99. Direct comparison of the mapping results presented here and the region around 87 is difficult due to a large inversion difference between $D$. melanogaster and $D$. sechellia in this region. The region around band 99 is not near this inversion. This region harbors 10 candidate ovariole genes (Wayne and McIntyre, 2002). Serr, which marked a region with a relatively strong effect on egg production, is in cytological band 97E. Thus, it is possible that one or more of the genes affecting the standing variation for ovariole number within $D$. melanogaster is involved in the interspecific difference in egg production. Of course, considerable additional work would be required to show that such a relationship exists.

\section{Acknowledgements}

I am grateful to J Gilman, B Stevenson, and AJ Tamer for technical assistance on this project. I also thank J Jaenike 
for good suggestions about the egg production assay. For excellent discussion and advice, I thank C Aquadro, A Betancourt, S Irving, LC Moyle, HA Orr, D Presgraves, and J Werren. I would also like to thank an anonymous reviewer for his/her helpful comments on the manuscript. This work was sponsored by grants from the David and Lucile Packard Foundation (HA Orr) and the National Institutes of Health (GM51932 to HA Orr) and by a Caspari Fellowship from the University of Rochester (CDJ) and the National Science Foundation (CDJ).

\section{References}

Adams MD, Celniker SE, Holt RA, Evans CA, Gocayne JD et al (2000). The genome sequence of Drosophila melanogaster. Science 287: 2185-2195.

Amlou M, Moreteau B, David JR (1998). Genetic analysis of Drosophila sechellia specialization: oviposition behavior toward the major aliphatic acids of its host plant. Behav Genet 28: 455-464.

Boulétreau-Merle J, Allemand R, Cohet Y, David JR (1982). Reproductive strategy in Drosophila melanogaster: significance of genetic divergence between temperate and tropical populations. Oecologia 53: 323-329.

Cariou ML, Solignac M, Monnerot M, David JR (1990). Low allozyme and mtDNA variability in the island endemic species Drosophila sechellia (D. melanogaster complex). Experientia 46: 101-104.

Coyne JA, Rux J, David JR (1991). Genetics of morphological differences and hybrid sterility between Drosophila sechellia and its relatives. Genet Res Camb 57: 113-122.

Coyne JA, Sniegowski PD (1994). A third-chromosome balancer in Drosophila simulans. Dros Inf Svc 75: 36-37.

David JR (1979). Utilization of morphological traits for the analysis of genetic variability in wild populations. Aquilo, Series Zoology 20: 49-61.

Farine JP, Legal L, Moreteau B, le Quere JL (1996). Volatile components of ripe fruits of Morinda citrifolia and their effects on Drosophila. Phytochemistry 41: 433-438.

Hodin J, Riddiford LM (2000). Different mechanisms underlie phenotypic plasticity and interspecific variation for a reproductive character in drosophilids (Insecta: Diptera). Evolution 54: 1638-1653.

Joly D, Bazin C, Zeng LW, Singh RS (1997). Genetic basis of sperm and testis length differences and epistatic effect on hybrid inviability and sperm motility between Drosophila simulans and D. sechellia. Heredity 78: 354-362.
Jones CD (1998). The genetic basis of Drosophila sechellia's resistance to a host plant toxin. Genetics 149: 1899-1908.

Jones CD (2001). The genetic basis of larval resistance to a host plant toxin in Drosophila sechellia. Genet Res Camb 78: 225-233.

Jones CD (2004). The genetics of adaptations in Drosophila sechellia. Genetica, in press.

Jones CD, Orr HA (1998). Remapping mutant markers on Drosophila simulans chromosome 3. Dros Inf Serv 81: 137-138.

Kampysellis MP, Heed WB (1971). Studies of oogenesis in natural populations of Drosophilidae. I. Relation of ovarian development and ecological habitats of Hawaiian species. Am Nat 105: 31-49.

Kliman RM, Andolfatto P, Coyne JA, Depaulis F, Kreitman M, Berry AJ et al (2000). The population genetics of the origin and divergence of the Drosophila simulans complex species. Genetics 156: 1913-1931.

Lachaise D, Cariou M-L, David R, Lemeunier F, Tsacas L, Ashburner M (1988). Historical biogeography of the Drosophila melanogaster species subgroup. Evol Biol 22: 159-226.

Louis J, David JR (1986). Ecological specialization in the Drosophila melanogaster species subgroup: a case study of D. sechellia. Acta Oecologica 7: 215-229.

R'Kha S, Capy P, David JR (1991). Host-plant specialization in the Drosophila melanogaster species complex: a physiological, behavioral, and genetical analysis. Proc Natl Acad Sci USA 88: 1835-1839.

R'Kha S, Moreteau B, Coyne JA, David JR (1997). Evolution of a lesser fitness trait, egg production in the specialist Drosophila sechellia. Genet Res Camb 69: 17-23.

Sucena E, Stern DL (2000). Divergence of larval morphology between Drosophila sechellia and its sibling species caused by cis-regulatory evolution of ovo/shaven-baby. Proc Natl Acad Sci USA 97: 4530-4534.

Wayne ML, Hackett JB, Dilda CL, Nuzhdin SV, Pasyukova EG, Mackay TFC (2001). Quantitative trait locus mapping of fitness-related traits in Drosophila melanogaster. Genet Res 77: 107-116.

Wayne ML, Hackett JB, Mackay TFC (1997). Quantitative genetics of ovariole number in Drosophila melanogaster. 1. Segregating variation and fitness. Evolution 51: $1156-1163$.

Wayne ML, Mackay TFC (1998). Quantitative genetics of ovariole number in Drosophila melanogaster. II. Mutational variation and genotype-environment interaction. Genetics 148: 201-210.

Wayne ML, McIntyre LM (2002). Combining mapping and arraying: an approach to candidate gene identification. Proc Natl Acad Sci USA 99: 14903-14906. 